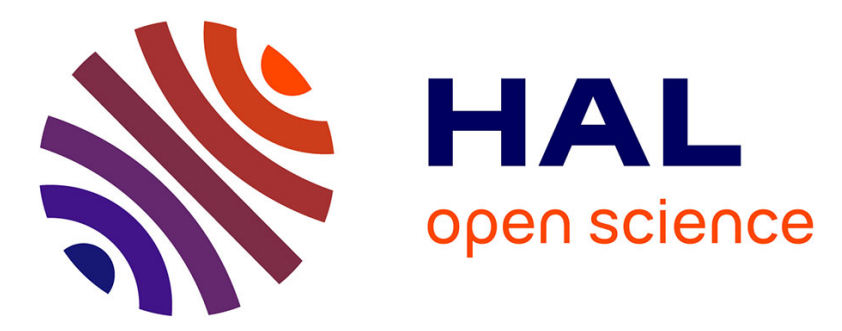

\title{
On extensions of HLLC scheme and its application to two-phase problems
}

Ksenia Kozhanova, Eric Goncalves, Yannick Hoarau, Philippe Parnaudeau

\section{To cite this version:}

Ksenia Kozhanova, Eric Goncalves, Yannick Hoarau, Philippe Parnaudeau. On extensions of HLLC scheme and its application to two-phase problems. 14th International Symposium on Numerical Analysis of Fluid Flows, Heat and Mass Transfer - Numerical Fluids Symposium 2019, ICNAAM 2019, 23-28 September 2019, Rhodes, Greece, Sep 2019, Rhodes, Greece. 10.1063/5.0026588 . hal03247241

\section{HAL Id: hal-03247241 \\ https://hal.science/hal-03247241}

Submitted on 2 Jun 2021

HAL is a multi-disciplinary open access archive for the deposit and dissemination of scientific research documents, whether they are published or not. The documents may come from teaching and research institutions in France or abroad, or from public or private research centers.
L'archive ouverte pluridisciplinaire HAL, est destinée au dépôt et à la diffusion de documents scientifiques de niveau recherche, publiés ou non, émanant des établissements d'enseignement et de recherche français ou étrangers, des laboratoires publics ou privés. 


\title{
On extensions of HLLC scheme and its application to two-phase problems
}

\author{
Ksenia Kozhanova $^{1}$, Eric Goncalves ${ }^{1}$, Yannick Hoarau ${ }^{2}$ and Philippe Parnaudeau ${ }^{1}$ \\ ${ }^{1}$ ISAE-ENSMA, Institut Pprime, UPR 3346 CNRS, Poitiers, France \\ ${ }^{2}$ Laboratoire ICube, University of Strasbourg, Strasbourg, France
}

\begin{abstract}
We study the numerical solution to two-phase problems using the reduced Kapila model, i.e. four-equations model. In particular we focus the present study on HLLC numerical flux approximation and its extensions. These numerical methods are tested on expansion tube cases with a final goal of constructing higher order hybrid numerical tool for solving the bubble collapsing under the shock wave problem.
\end{abstract}

\section{Introduction}

The importance of two-phase fluid flow modelling arises from many practical applications, from hydraulic turbines to power generation plants. However, the hyperbolic nature of the system describing such a flow and their characteristic analysis makes it extremely complicated task for applied mathematics and numerical methods. There are variety of different methods to solve this problem based on physical assumptions for the slip condition between the phases and mechanical and thermodynamic equilibriums. Yet, the most complete modelling approach is known to be two-fluid model which, however, as many other methods, has the challenge of dealing with spurious oscillations due to large variations of speed of sound and discontinuities of thermodynamic variables.

In this work we focus on four-equation model [1] and aim to establish the numerical scheme effect on the basis of inviscid applications, where we include three conservation laws for mixture quantities and void ratio for transport equation. The solver is based on the explicit finite volume methods using different schemes such as HLLC with different resolution approaches in time and limiting techniques in space.

\section{Mathematical model}

This section discusses the mathematical representation of the two-fluid model with homogeneous mixture approach. We solve the problem under the assumptions of well mixed phases and small size of disperse particles, which allow the elimination of relative motion, strong coupling of phases with the same velocity, thermal and mechanical equilibrium of the phases and saturation state of liquid.

The present four-equation model consists of three conservation laws for mixture quantities and fourth equation for void-ratio [1]. Particularly, the void ratio $\alpha$ equation states,

$$
\frac{\partial \alpha}{\partial t}+\vec{V} \cdot \operatorname{grad}(\alpha)=K \operatorname{div}(\vec{V}), \text { with } K=\frac{\rho_{l} c_{l}^{2}-\rho_{v} c_{v}^{2}}{\frac{\rho_{l} c_{l}^{2}}{1-\alpha}+\frac{\rho_{v} c_{v}^{2}}{\alpha}}
$$

where $\vec{V}=(u, v)$ denotes the center of mass velocity. The reflection of the change in each phase volume and speed of sound of pure phases $c_{k}$ are included into the term $K$. The equations of states (EOS) are used to relate pressure and temperature to internal energy and density to close the system,

$$
P(\rho e)=(\Gamma-1) \rho(e-q)-\Gamma P_{\infty}, P(\rho T)=\rho(\Gamma-1) C_{v} T-P_{\infty}, T(\rho h)=\frac{h-q}{C_{p}}
$$

where $C_{p}$ and $C_{v}$ are thermal capacities, $q$ is the energy of formation and $\Gamma=C_{p} / C_{v}$ is the heat capacity ratio. $P_{\infty}$ stands for constant reference pressure. 
Using the finite volume method, the discretization of computational spatial and temporal domains into regular meshes of some length $\Delta x$ and intervals $\Delta t$, respectively, can be performed. The system, thus, is reformulated into its discrete form in the following manner,

$$
\Delta x \frac{w_{i}^{n+1}-w_{i}^{n}}{\Delta t}+F_{i+1 / 2}^{n}-F_{i-1 / 2}^{n}=S_{i}^{n} \Delta x
$$

where $S(w)$ is the source term and $w$ is the conservative variables set. We are looking to approximate the numerical flux $F_{i+1 / 2}^{n}$, $F_{i-1 / 2}^{n}$ using the solution to Riemann problem or any other fully numerically resolved technique. This report studies HLLC flux approximation with different extensions, while quite a large amount of other numerical approximations are available. HLLC method provides the first order in space tool, which can be improved using different numerical tools, which are discussed in the coming sections.

\section{Numerical methods}

MUSCL reconstruction can be applied to any set of variables one prefers to work with. However, it has been known that the primitive variables is a better less-oscillating choice [2]. Saying that, we denote some primitive interface variable we would like to reconstruct, $w_{\frac{1}{2}}^{L}$, the reconstruction parameter, $\phi$, which can chosen to be 1 or -1 to achieve the second order decentered or centered scheme, respectively, or $\frac{1}{3}$ for third order in space scheme, and the TVD slope limiter operator of the choice, $\Psi$. MUSCL extrapolation can be formulated as follows,

$$
w_{\frac{1}{2}}^{L}=w_{i}+\frac{1-\phi}{4} \Psi\left(r^{L}\right)\left(w_{i}-w_{i-1}\right)+\frac{1+\phi}{4} \Psi\left(\frac{1}{r^{L}}\right)\left(w_{i+1}-w_{i}\right) \text { with } r^{L}=\frac{w_{i+1}-w_{i}}{w_{i}-w_{i-1}}
$$

Particularly, VanAlbada limiter is $\Psi(r)=\max \left(0, \frac{r+r^{2}}{1+r^{2}}\right)$.

HLLC MUSCL might be combined with MH [2] scheme by performing reconstruction part with advancing the solution by half time step before the derivation of time-centered interface values, i.e.

$$
\tilde{\mathbf{W}}_{j}=\mathbf{W}_{j}-\frac{\Delta t}{2 \Delta x} \mathbf{A}_{W} \delta \mathbf{W}_{j}
$$

where $\mathbf{A}_{W}$ is a matrix, which depends on the choice of set of variables (e.g. primitive variables would require the primitive form of equations, while conservative set of variables needs the derivation of Jacobian matrix, which is fairly complicated in current problem formulation).

Furthermore, to achieve the higher order in space scheme, we introduce the PPM slope limiter [3] by using the extrapolation under the parabola, i.e.

$$
w_{i}^{L}=\frac{7}{12}\left(w_{i}+w_{i+1}\right)-\frac{1}{12}\left(w_{i+2}+w_{i-1}\right)
$$

We work with PPM method using the monotonicity algorithm with or without narrow profile, which allows steeper discontinuity representation, depending on the considered problem.

\section{Numerical tests and results}

We validate presented numerical methods using the problems of expansion tube with low and high velocity profiles and shockbubble collapse. These tests have been performed in hierarchical order in terms of the stiffness of the considered problem. The first problem in consideration is double rarefaction one meter long tube with velocity initialised with discontinuity located in the middle. The tube filled with liquid water with density $\rho_{l}=1150 \mathrm{~kg} / \mathrm{m}^{3}$, atmospheric pressure and fraction of vapor $\alpha=0.01$. The initial velocity is set to be $-2 \mathrm{~m} / \mathrm{s}$ on the left and $2 \mathrm{~m} / \mathrm{s}$ on the right.

We note, that pressure should ensured to be positive since gas is present. In order to do so, we introduce an increase in gas volume fraction using the gas mechanical expansion. The obtained numerical solution has two expansion waves, which corresponds to the physical nature of the test and has been modelled using 1000 cells at time step equal to $10^{-7} \mathrm{~s}$. We compare the results at final time $T=3.2 \mathrm{~ms}$.

The plots on Fig. 1 have been obtained applying the following numerical techniques:

- $\quad$ MUSCL Hancock scheme with Van Albada slope limiter

- $\quad$ PPM with third order in time Runge-Kutta resolution (RK3)

- $\quad$ PPM combined with MUSCL Hancock

- $\quad$ third order MUSCL extrapolation with Van Albada slope limiter (not presented on the graph). 

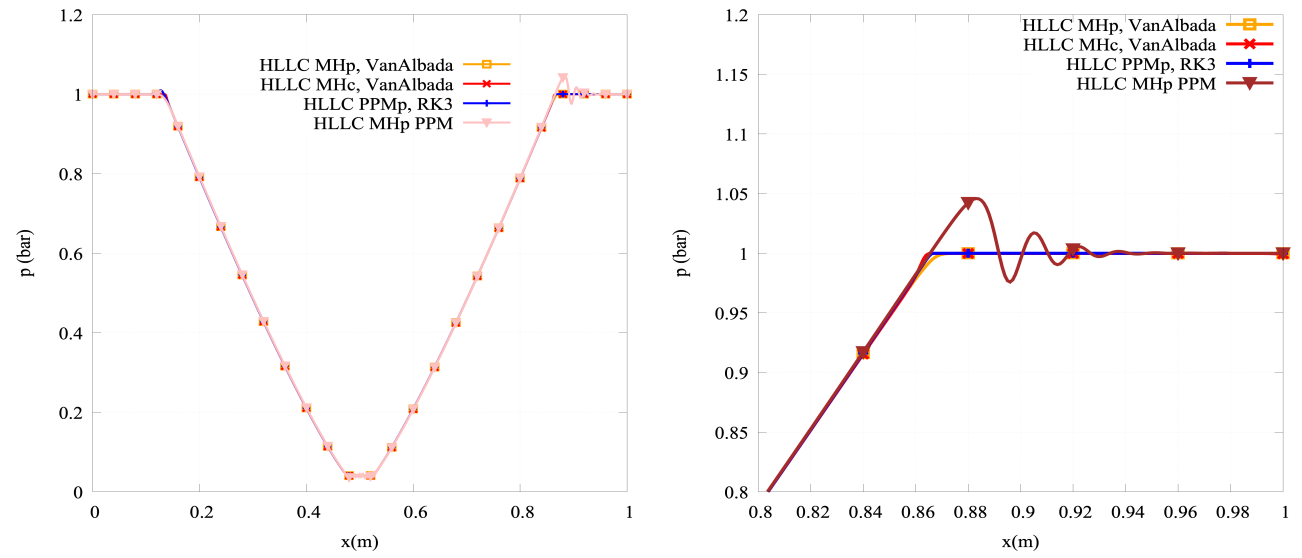

FIGURE 1. Expansion tube, $\left|u_{0}\right|=2 \mathrm{~m} / \mathrm{s}, \mathrm{T}=3.2 \mathrm{~ms}$

It comes immediately, that both, PPM and PPM with Hancock's method leads to higher non symmetrical oscillations. Enlarging the graph between 0.8 and 1 in abscissa, we observe the sharpest representation using PPM with third order Runge-Kutta with Hancock's method almost matching this result.

Next test is similar to the previous one except for the velocity value which we increase to $100 \mathrm{~m} / \mathrm{s}$ which results in stronger velocity discontinuity. As before, we set $d t=10^{-7} \mathrm{~s}$ and perform the computations on the domain with 1000 cells. The solutions have been compared at final time $T=1.5 \mathrm{~ms}$.

The higher velocity profile results in numerically stiffer problem and we expect the reduced performance of some schemes. Saying that, we do not obtain the results for MH scheme coupled with PPM narrow profile. However, we successfully run this test with switching off narrow profiling for pressure, (Fig. 2). Furthermore, we observe the performance of PPM with and without narrow profile trick: indeed, the representation using narrow profiling is the sharpest we see for this problem. Notably, using simple parabolic extrapolation for pressure allowed us to significantly reduce the left side oscillation due to the local order reduction. However, as previously seen, all types of PPM demonstrate the oscillation on the left. On the other hand, MUSCL Hancock with primitive variables provides non-oscillating curve, while conservative variables extrapolation leads to high peaks as the wave expands. Lastly, HLLC scheme with third order in space MUSCL extrapolation demonstrates the highest oscillations, which are generally expected with higher order schemes.
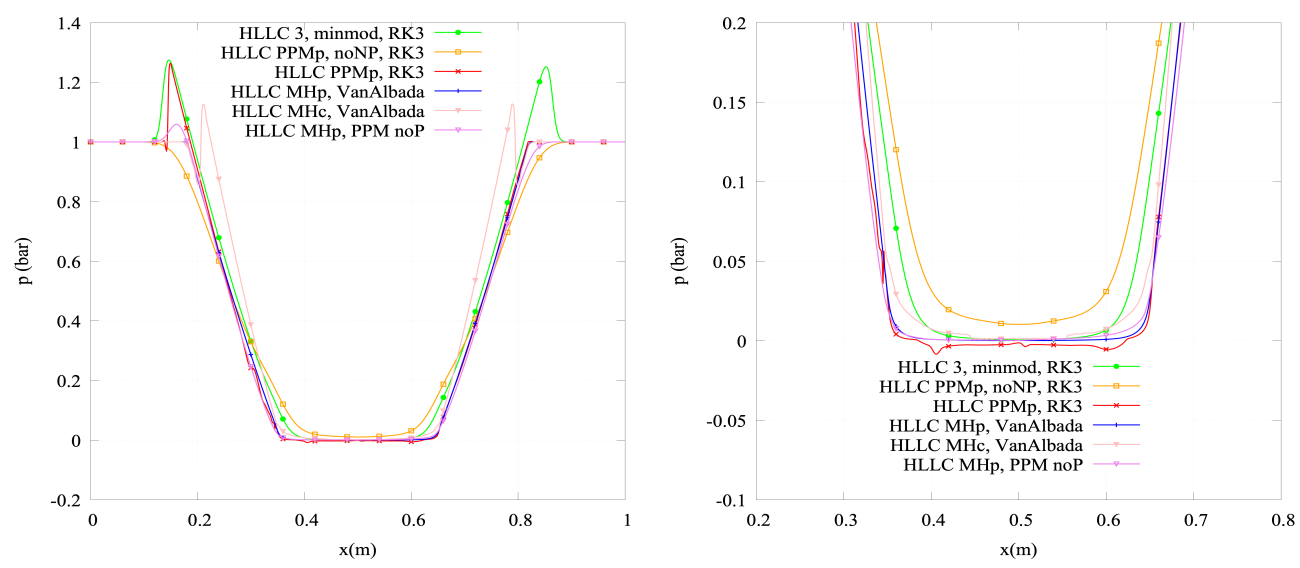

FIGURE 2. Expansion tube, $\left|u_{0}\right|=100 \mathrm{~m} / \mathrm{s}, \mathrm{T}=1.5 \mathrm{~ms}$

The final test we perform is describing the air bubble of cylindrical form with diameter $6 \mathrm{~mm}$ inside the water with weak volume fraction of gas $\alpha_{0}=10^{-10}$. The physical conditions are initialised as following: $\vec{V}=(0,0) \mathrm{m} / \mathrm{s}, P=10^{5} \mathrm{~Pa}, \rho_{\text {air }}=1 \mathrm{~kg} / \mathrm{m}^{3}$ and $\rho_{\text {water }}=1000 \mathrm{~kg} / \mathrm{m}^{3}$. The bubble's location is inside the domain of size $12 \times 24 \mathrm{~mm}$ with center of the bubble located at $(9,6)$ $\mathrm{mm}$, while the wave moves with the speed $M_{s h}=1.72$ leading to eventual bubble collapse. The domain has top and bottom boundaries to be set as walls, and left and right boundaries non-reflective conditions. The computations have been made with spacial discretization of $800 \times 400$ cells using $d t=10^{-9} \mathrm{~s}$. 
The bubble collapse problem is known to be fairly difficult in terms finding the stable higher order non-oscillating numerical scheme. Our tests demonstrated good numerical result using HLLC with MH primitive variables extrapolation, while PPM without narrow profile provided considerably less curve reconstruction. On the other hand, narrow profile technique applied to PPM does not work in this test, unless the pressure reconstruction is reduced to simple parabolic computation and time step decreased to $10^{-10}$ s. However, even this reduction does not lead to the results comparable with MH primitive scheme. Additionally, all results for this problem have been obtained using first order extrapolation for density. Fig. 3 presents the density gradient and pressure profiles at $\mathrm{T}=5.6 \mathrm{~ms}$ (on the left top and bottom, respectively) using HLLC MH primitive scheme, and max pressure profile (on the right) at $\mathrm{T}=6 \mathrm{~ms}$. The first peak in max presure can be observed at time $\mathrm{t}=3.8 \mathrm{~m} / \mathrm{s}$ and is being best captured by MUSCL Hancock scheme with primitive extrapolation (green line with circles), while PPM without narrow profile (orange line with triangles) provides considerably less reconstruction. In regard to PPM with narrow profile option (pink line with squares), we notice fairly good capture of the peak, yet, with high oscillations. The second peak in max pressure occurs at approximately $\mathrm{t}=4.7 \mathrm{~ms}$ and, once more, MH with primitive variables led to the best outcome, while both PPM schemes provided less curve information. Provisionally, we believe that the middle ground scheme for stiff cases might be HLLC MH scheme with primitive reconstruction, while PPM limiter can potentially provide higher order results, but should be examined further, especially on the subject of narrow profiling method.
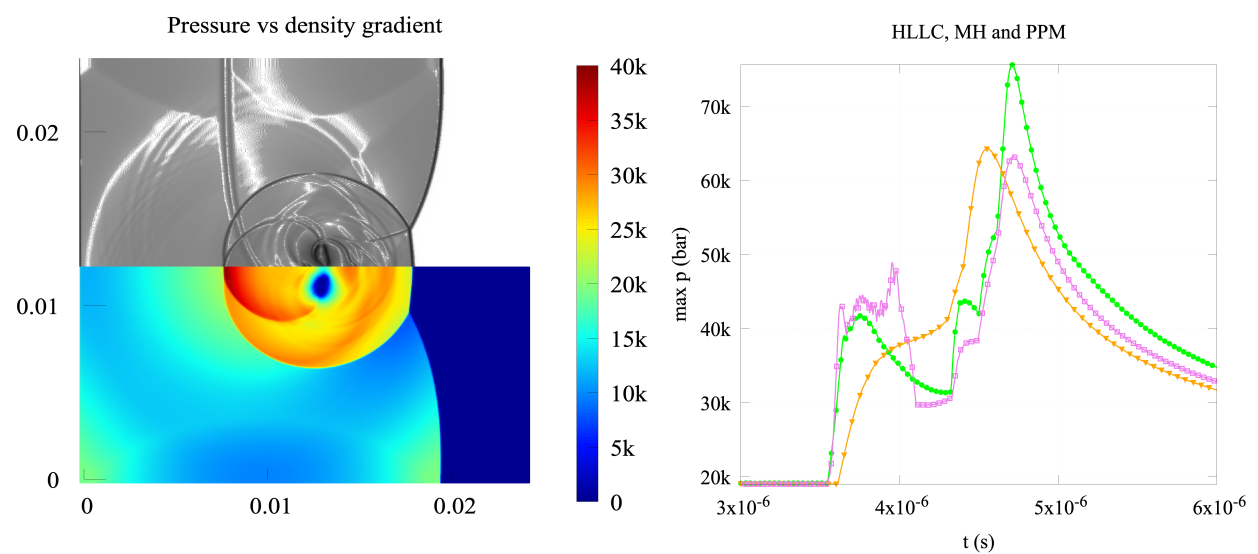

FIGURE 3. Bubble collapse

\section{Conclusion}

The present work has been focusing on HLLC flux approximation with its different extensions for improving the accuracy of the overall solution. We implemented and tested several slope limiters in the framework of MUSCL extrapolation, such as minmod, superbee and Van Albada. MUSCL extrapolation has been further coupled with Hancock's predictor-corrector scheme using both, primitive and conservative sets of variables. Additionally, we introduce PPM slope limiting technique with and without narrow profile method. We tested all the numerical tools on variety of physical problems in hierarchical order in terms of the stiffness of the hyperbolic system. We believe that our results demonstrated the fair stability of MH scheme with primitive variables, while PPM method should be considered further, as potentially higher order reconstruction, yet with some additional oscillations limiter.

\section{Acknowledgements}

This work has been partially supported by French ANR (ANR-18-CE46-0009).

\section{REFERENCES}

[1] E. Goncalves and D. Zeidan, "Simulation of Compressible Two-Phase Flows Using a Void Ratio Transport Equation", Commun. Comput. Phys., 24(1), pp.167-203, (2018).

[2] B. Leer, "Upwind and high-resolution methods for compressible flow: from donor cell to residual-distribution schemes", 16th AIAA Computational Fluid Dynamics Conference, (2003).

[3] P. Colella, P.R. Woodward, "The piecewise parabolic method (PPM) for gas-dynamical simulations", Journal of Computational Physics, 54(1), pp. 174-1984, (1984). 